\title{
Non-thermal plasma specifically kills oral squamous cell carcinoma cells in a catalytic Fe(II)-dependent manner
}

\author{
Kotaro Sato, ${ }^{1,2}$ Lei Shi, ${ }^{1}$ Fumiya Ito, ${ }^{1}$ Yuuki Ohara, ${ }^{1}$ Yashiro Motooka, ${ }^{1}$ Hiromasa Tanaka, ${ }^{3}$ Masaaki Mizuno, ${ }^{3}$ \\ Masaru Hori, ${ }^{4}$ Tasuku Hirayama, ${ }^{5}$ Hideharu Hibi ${ }^{2}$ and Shinya Toyokuni ${ }^{1,6, *}$
}

\author{
'Department of Pathology and Biological Responses and ${ }^{2}$ Department of Oral and Maxillofacial Surgery, Nagoya University Graduate School of Medicine, \\ 65 Tsurumai-cho, Showa-ku, Nagoya 466-8550, Japan \\ ${ }^{3}$ Center for Advanced Medicine and Clinical Research, Nagoya University Hospital, Nagoya 466-8550, Japan \\ ${ }^{4}$ Plasma Nanotechnology Research Center, Nagoya University, Nagoya 464-8603, Japan \\ ${ }^{5}$ The Laboratory of Pharmaceutical and Medicinal Chemistry, Gifu Pharmaceutical University, Gifu 501-1196, Japan \\ ${ }^{6}$ Sydney Medical School, The University of Sydney, NSW 2006, Australia
}

(Received 29 September, 2018; Accepted 28 January, 2019; Published online 1 June, 2019)

\begin{abstract}
Oral cancer accounts for $\sim 2 \%$ of all cancers worldwide, and therapeutic intervention is closely associated with quality of life. Here, we evaluated the effects of non-thermal plasma on oral squamous cell carcinoma cells with special reference to catalytic Fe(II). Non-thermal plasma exerted a specific killing effect on oral squamous cell carcinoma cells in comparison to fibroblasts. Furthermore, the effect was dependent on the amounts of catalytic Fe(II), present especially in lysosomes. After non-thermal plasma application, lipid peroxidation occurred and peroxides and mitochondrial superoxide were generated. Cancer cell death by non-thermal plasma was promoted dose-dependently by prior application of ferric ammonium citrate and prevented by desferrioxamine, suggesting the association of ferroptosis. Potential involvement of apoptosis was also observed with positive terminal deoxynucleaotidyl transferase-mediated dUTP nick end labeling and annexin $V$ results. Non-thermal plasma exposure significantly suppressed the migratory, invasive and colony-forming abilities of squamous cell carcinoma cells. The oral cavity is easily observable; therefore, non-thermal plasma can be directly applied to the oral cavity to kill oral squamous cell carcinoma without damaging fibroblasts. In conclusion, non-thermal plasma treatment is a potential therapeutic option for oral cancer.
\end{abstract}

Key Words: non-thermal plasma, oral squamous cell carcinoma, catalytic ferrous iron, apoptosis, ferroptosis

$\mathrm{O}$ ral cancer accounts for approximately $2 \%$ of all cancers worldwide, but the proportion has gradually increased in some countries. ${ }^{(1)}$ Smoking, alcohol abuse, use of smokeless tobacco products and HPV infections are listed as high-risk factors for oral cancer. ${ }^{(2-4)}$ As oral cancer develops and infiltrates into the surrounding tissues with accompanying necrosis, it causes severe pain, nerve damage and facial deformation and is directly linked to a decrease in quality of life. Therefore, early detection and treatment are very important, as in other cancers. In addition, monitoring for locoregional recurrence and distant metastasis after surgery is also important.

Plasma is the fourth physical state beyond the normal solid/ liquid/gas phases and is a mixture of gas, radicals, electrons, cations, anions, and ultraviolet (UV) radiation. ${ }^{(5,6)}$ Non-thermal plasma (NTP) represents an apparently contradictory condition with high energy but at near-body temperature, which was realized through engineering in the 1990's. NTP is currently applied in preclinical experiments in a variety of fields, especially in the medical field. ${ }^{(7-10)}$
Studies have shown that NTP acts specifically on cancer cells, leading to cell death, and therefore represents a notable new potential cancer treatment. ${ }^{(11-14)}$ NTP has the same temperature as body temperature, and many chemical species/agents have been detected with NTP exposure, including ${ }^{\circ} \mathrm{OH}, \mathrm{H}_{2} \mathrm{O}_{2}, \mathrm{O}_{2}^{-}$, nitric oxide, electrons and UV radiation. ${ }^{(15)}$ Sufficient evidence demonstrates that NTP promotes wound healing and possibly kills cancer cells. ${ }^{(13,16-18)}$ The theoretical basis for NTP use in cancer treatment is that cancer cells are oxidatively stressed in general, and the Fenton reaction occurs after NTP exposure. ${ }^{(19)}$

We have thus far conducted various research projects on the relationship between excess iron and carcinogenesis ${ }^{(20-25)}$ and recently showed that iron chelation or phlebotomy by reducing local iron can suppress mesothelial carcinogenesis. ${ }^{(26,27)}$ A phlebotomy intervention trial in humans led to not only a decreased cancer incidence but also significantly decreased cancer mortality; ${ }^{(28)}$ thus, a close relationship exists between cancer and iron. Although the effect of NTP on oral cancer cells has previously been reported, ${ }^{(29,30)}$ research has not been performed to explore the relationship between oral cancer cells and iron metabolism. Thus, the objective of this study was to evaluate the effect of NTP on oral cancer cells and its association with catalytic Fe(II).

\section{Materials and Methods}

Cell lines and reagents. We used seven human oral squamous cell carcinoma cell lines (SAS, Ca9-22, HSC-2, HSC-3, HSC-4, Sa3 and Ho-1-u-1) and two human fibroblast cell lines (HS-K and IMR-90-SV) in this study. The oral squamous cell carcinoma cells were all obtained from the Riken Cell Bank (Ibaraki, Japan). The fibroblast cell lines were also obtained from the Riken Cell Bank and were used as a control. All the cells were grown in RPMI-1640 medium (Wako, Osaka, Japan) containing $10 \%$ fetal bovine serum (FBS) (Biowest, Nuaillé, France) and 1\% antibiotic-antimycotic solution (Invitrogen, Carlsbad, CA), hereafter referred to as complete medium, and $0.25 \%$ trypsin (Nacalai Tesque, Kyoto, Japan) was used to collect cells. The cells were maintained at $37^{\circ} \mathrm{C}$ in a humidified incubator with $5 \% \mathrm{CO}_{2}$.

Non-thermal plasma (NTP). NTP was generated by the following well-established procedure ${ }^{(17)}$ using a Habahiro (wide orifice) instrument (from Prof. M. Hori, Plasma Nanotechnology

*To whom correspondence should be addressed. E-mail: toyokuni@med.nagoya-u.ac.jp 
Research Center, Nagoya University, Nagoya, Japan) by applying $10 \mathrm{kV}$ from a $60-\mathrm{Hz}$ commercial power supply, using 2 electrodes that were $20 \mathrm{~mm}$ apart. The NTP had an ultrahigh electron density (approximately $2 \times 10^{16} \mathrm{~cm}^{3}$ ) and an $\mathrm{O}$ density of approximately $4 \times 10^{15} \mathrm{~cm}^{3}$, as previously reported. ${ }^{(31)}$ Argon (Ar) was used as the gas to generate NTP and was implemented at a flow rate of $2 \mathrm{~L} / \mathrm{min}$. The distance between the plasma source and the samples was fixed at $\mathrm{L}=8 \mathrm{~mm}$. In all the experiments, cells in complete media were treated with NTP for $30-120 \mathrm{~s}$ at $25^{\circ} \mathrm{C}$ and then returned to $37^{\circ} \mathrm{C}$ for various incubation periods. For studies using 96-well plates, a cover was placed to expose only one well, as previously reported. ${ }^{(31)}$ As a relevant control, studies were performed using Ar alone under the same experimental conditions.

Cell viability assay. Nine kinds of cell lines were cultured in 96-well plates (Thermo Fisher Scientific, Waltham, MA) at 5,000 cells/well for $24 \mathrm{~h}$ at $37^{\circ} \mathrm{C}$. NTP exposure $(30,60,90$, and $120 \mathrm{~s}$ at $25^{\circ} \mathrm{C}$ ) or $\mathrm{Ar}$ gas only was applied as a control to the cells, which were then cultured for $24 \mathrm{~h}$ at $37^{\circ} \mathrm{C}$. Absorbance was measured at $450 \mathrm{~nm}$ using the WST- 8 cell proliferation assay (Nacalai Tesque) and the POWERSCAN 4 microtiter plate reader (BioTek, Winooski, VT) to determine the viability of the cells after the treatment.

Annexin V-fluorescein isothiocyanate (FITC) and propidium iodide (PI) analyses and terminal deoxynucleotidyl transferasemediated dUTP nick end labeling (TUNEL) assay. We selected two squamous cell carcinoma cell lines (SAS and Ca9-22) that were highly sensitive to NTP and one non-tumorous fibroblast line (IMR-90-SV). In the NTP group and the Ar alone group, a total of $1.5 \times 10^{6}$ cells/well were seeded in a $60-\mathrm{mm}$ dish (Corning, Corning, NY) and cultured for $24 \mathrm{~h}$. NTP or Ar alone was applied, and the cells were incubated for an additional $24 \mathrm{~h}$. After incubation, we used the Annexin V-FITC Apoptosis Detection Kit according to the manufacturer's protocol (Nacalai Tesque) and evaluated cell death using the Gallios flow cytometer (Beckman Coulter, Brea, CA). Furthermore, we performed a TUNEL assay. In the NTP group and the Ar alone group, a total of 5,000 cells/ well were seeded in a 96-well plate (Thermo Fisher Scientific) and cultured for $24 \mathrm{~h}$. NTP or Ar alone was applied, and the cells were incubated for another $24 \mathrm{~h}$. After incubation, we used the In Situ Cell Death Detection Kit, Fluorescein to observe apoptosis microscopically according to the manufacturer's protocol (Roche Applied Science, Mannheim, Germany) and observed the staining results, using the BZ9000 microscope (Keyence, Osaka, Japan).

Quantitation and localization of catalytic Fe(II).

SiRhoNox-1, which is a catalytic Fe(II)-specific probe, was synthesized and characterized as previously described ${ }^{(32)}$ and was adjusted to $1 \mu \mathrm{M}$ in RPMI-1640 without phenol red (Wako). Then, $5 \times 10^{5}$ cells/well were seeded in a $60-\mathrm{mm}$ dish and cultured for $24 \mathrm{~h}$. After incubation, the cells were stained with SiRhoNox-1 $(1 \mu \mathrm{M})$ for $30 \mathrm{~min}$ and washed with phosphate-buffered saline (PBS) (Wako). The fluorescence intensity of each cell was evaluated using a Gallios flow cytometer. Subsequently, $2.5 \times 10^{5}$ cells were plated into glass-bottom dishes (Matunami, Osaka, Japan) and incubated overnight. Then, the cells were stained with SiRhoNox-1 $(1 \mu \mathrm{M})$, MitoTracker Green FM (50 nM: Thermo Fisher Scientific) and ER-Tracker Blue-White DPX (200 nM: Thermo Fisher Scientific) for $30 \mathrm{~min}$ and LysoTracker Red DND-99 (200 nM: Thermo Fisher Scientific) for $60 \mathrm{~min}$. The cells were then washed twice with PBS and observed using a Zeiss confocal microscope LSM880 (Carl Zeiss, Oberkochen, Germany). The fluorescence intensity for localization was evaluated, using Image J 4.7v software (National Institutes of Health, Bethesda, MD).

Effects of ferric ammonium citrate (FAC) and desferrioxamine (DFO) administration prior to NTP application. SAS and $\mathrm{Ca} 9-22$ cells were seeded in $60-\mathrm{mm}$ dishes at $5.0 \times 10^{5}$ cells for $24 \mathrm{~h}$. Ferric ammonium citrate (FAC) $(10 \mu \mathrm{g} / \mathrm{ml}$ : SigmaAldrich, St. Louis, MO), which is a well-characterized cellular $\mathrm{Fe}$ donor, was dissolved in milli-Q water, and the cells were loaded for $3 \mathrm{~h}$ with FAC. Then, the culture medium was exchanged and stained with SiRhoNox-1 $(1 \mu \mathrm{M})$ for $30 \mathrm{~min}$ and washed twice with PBS. Desferrioxamine (DFO) $(25 \mu \mathrm{M}$ : SigmaAldrich), which is known to decrease cellular iron levels, was dissolved in dimethyl sulfoxide, and the cells were loaded for $3 \mathrm{~h}$ with DFO. Then, the culture medium was exchanged and stained with SiRhoNox-1 $(1 \mu \mathrm{M})$ for $30 \mathrm{~min}$ and washed twice with PBS. The fluorescence intensity of each cell was analyzed, using Gallios flow cytometry. Subsequently, SAS and Ca9-22 cells were cultured in 96-well plates at 5,000 cells/well for $24 \mathrm{~h}$ at $37^{\circ} \mathrm{C}$. FAC was loaded into the cells for $3 \mathrm{~h}$, and then the culture medium was exchanged. NTP was applied for $30 \mathrm{~s}$, and the cells were cultured for $24 \mathrm{~h}$ at $37^{\circ} \mathrm{C}$. DFO was also loaded into the cells for $3 \mathrm{~h}$. Then, the culture medium was exchanged, NTP was applied for $90 \mathrm{~s}$, and the cells were cultured for $24 \mathrm{~h}$ at $37^{\circ} \mathrm{C}$. Absorbance was measured at $450 \mathrm{~nm}$, using the WST-8 cell proliferation assay and POWERSCAN 4 microtiter plate reader to determine the viability of the cells after application.

Analysis of reactive oxygen species (ROS) generation after applying NTP. In the NTP group and the Ar alone group, a total of $5.0 \times 10^{5}$ cells/well were seeded in a $60-\mathrm{mm}$ dish and cultured for $24 \mathrm{~h}$. We removed the medium and added plain RPMI (FBS-, ANTI-). Then, NTP or Ar alone was applied for $60 \mathrm{~s}$, followed by incubation for $30 \mathrm{~min}$. We stained the cells with MitoSOX (5 $\mu \mathrm{M}$ : Thermo Fisher Scientific) and a chloromethyl derivative of 2',7'-dichlorodihydrofluorescein (DCF) $(10 \mu \mathrm{M}$ : Thermo Fisher Scientific) in either RPMI-1640 without phenol red for $10 \mathrm{~min}$ or BODIPY 581/591 C11 (BODIPY) $(2 \mu \mathrm{M}$ : Thermo Fisher Scientific) in RPMI-1640 without phenol red for $15 \mathrm{~min}$. After washing twice with PBS, we evaluated ROS, using the Gallios flow cytometer.

Scratch wound-healing assay. Cells were seeded in 35-mm dishes (Iwaki, Shizuoka, Japan) and cultured to $100 \%$ confluence. Scratches were made with $200-\mu 1$ pipette tips, and the cells were photographed with a phase contrast microscope. Then, the Ar alone group and the NTP group were examined (60 s). After $24 \mathrm{~h}$, we observed the cells with a phase contrast microscope.

Transwell migration assay. In the NTP group and the Ar alone group, a total of $1.0 \times 10^{6}$ cells/well were seeded in a $60-\mathrm{mm}$ dish and cultured for $24 \mathrm{~h}$. NTP or Ar alone was applied, and the cells were incubated for $30 \mathrm{~min}$. Then, a cell culture insert (Corning) was placed in a 24 -well plate, and cells were seeded at $5.0 \times 10^{5}$ cells $/ 200 \mu \mathrm{l}$ in the same well. After $24 \mathrm{~h}$, the cells were stained with hematoxylin and counted.

Transwell invasion assay. In the NTP group and the Ar alone group, a total of $1.0 \times 10^{6}$ cells/well were seeded in a $60 \mathrm{~mm}$ dish and cultured for $24 \mathrm{~h}$. After incubation, NTP or Ar alone was applied, and the cells were incubated for $30 \mathrm{~min}$. Then, Matrigel matrix (Corning) was added to a cell culture insert, which was placed in a 24-well plate, and the cells were seeded at $5.0 \times 10^{5}$ cells $/ 200 \mu \mathrm{l}$, as previously reported. ${ }^{(33)}$ After $24 \mathrm{~h}$, the cells were stained with hematoxylin and counted.

Western blotting. For the treatments with NTP and Ar alone, a total of $1.0 \times 10^{6}$ cells/well were seeded in a $60-\mathrm{mm}$ dish and cultured for $24 \mathrm{~h}$. After incubation, NTP or Ar alone was applied, and the cells were incubated for $24 \mathrm{~h}$. Protein extraction and sodium dodecyl sulfate-polyacrylamide gel electrophoresis were performed, as previously described, ${ }^{(34)}$ using anti-matrix metalloproteinase-2 (MMP-2) rabbit polyclonal antibody (Cell Signaling Technology, G657; Danvers, MA) at 1:1,000 dilution.

Soft agar colony formation assay. In the NTP group and the Ar alone group, a total of $1.0 \times 10^{6}$ cells/well were seeded in 6 -well plates (Corning) and cultured for $24 \mathrm{~h}$. After incubation, NTP or Ar alone was applied, and the cells were incubated for 30 min. During incubation, we prepared agar, using Agar Noble (Becton, Dickinson and Company, Franklin Lakes, NJ). We poured $2.5 \mathrm{ml}$ of $0.75 \%$ agar into the 6 -well plate as bottom agar. After confirming that the bottom agar had solidified, we collected 

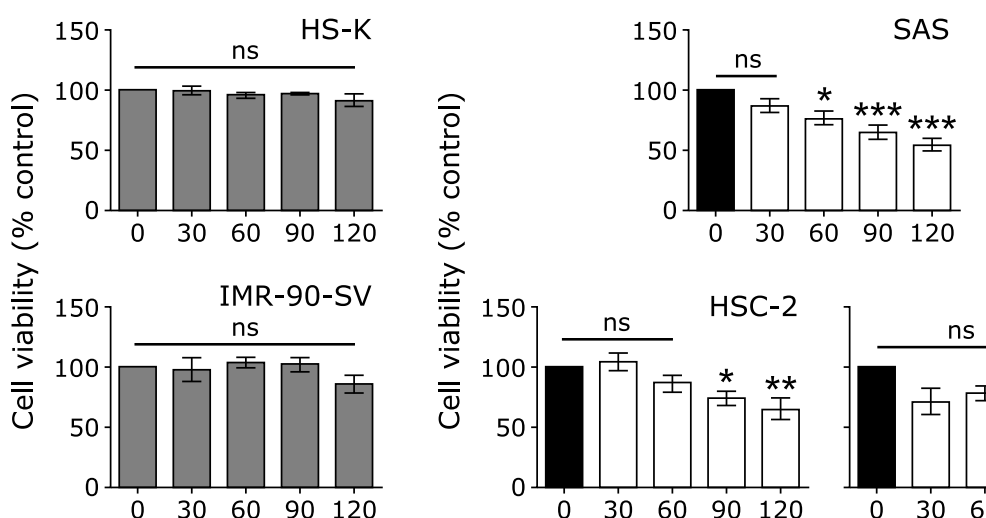

Oral squamous cell carcinoma cell lines

Non-thermal plasma treatment time $(\mathrm{s})$

B

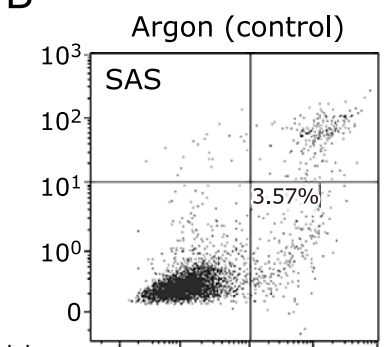

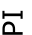

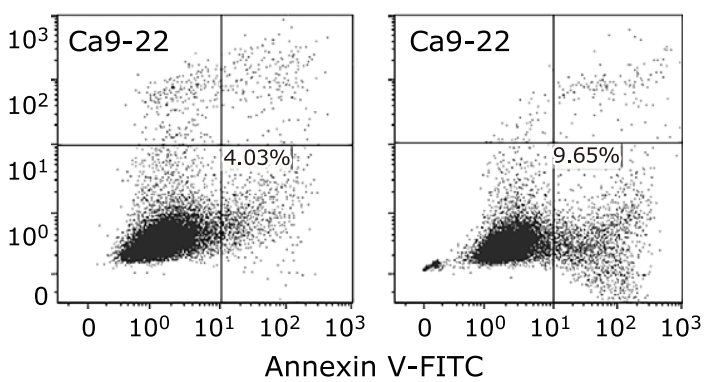

Non-thermal plasma $\quad$ C
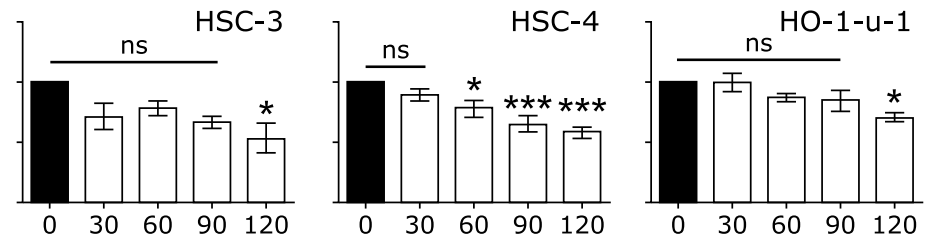

Non-thermal plasma treatment time (s)

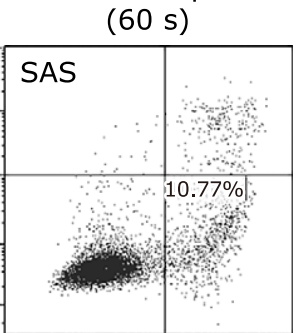

Argon (control)

Non-thermal plasma (60 s)

Phase contrast Merged Phase contrast Merged

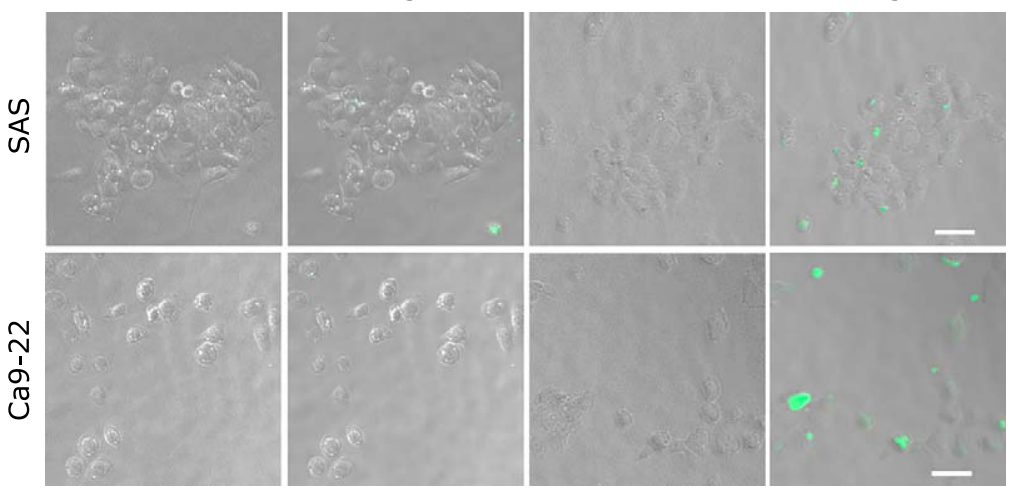

Fig. 1. Cell viability assay and detection of apoptosis. (A) Cell viability assay. Nine cell lines were cultured in 96 -well plates at 5,000 cells/well for $24 \mathrm{~h}$ at $37^{\circ} \mathrm{C}$. Non-thermal plasma (NTP) $\left(30,60,90\right.$, and $120 \mathrm{~s}$ at $\left.25^{\circ} \mathrm{C}\right)$ was applied, Argon (Ar) alone was applied for $60 \mathrm{~s}$ to the control cells, and the cells were cultured for $24 \mathrm{~h}$ at $37^{\circ} \mathrm{C}$. Absorbance was measured using the WST-8 cell proliferation assay and POWERSCAN 4 microtiter plate reader. Error bars represent the standard error of the mean. ${ }^{*} p<0.05, * * p<0.01, * * * p<0.001$; ns, not significant. (B) Annexin V-fluorescein isothiocyanate (FITC) and propidium iodide (PI) analysis. In the NTP group and the Ar alone group, a total of $1.5 \times 10^{6}$ cells/well were seeded in a $60-\mathrm{mm}$ dish and cultured for $24 \mathrm{~h}$ at $37^{\circ} \mathrm{C}$. NTP or Ar alone was applied, and cells were incubated for $24 \mathrm{~h}$ at $37^{\circ} \mathrm{C}$. After incubation, apoptosis was measured using flow cytometry. (C) Terminal deoxynucleotidyl transferase-mediated dUTP nick end labeling (TUNEL) assay. In the NTP group and the Ar alone group, a total of $5.0 \times 10^{3}$ cells/well were seeded in a 96-well plate and cultured for $24 \mathrm{~h}$ at $37^{\circ} \mathrm{C}$. NTP or Ar alone was applied, and cells were incubated for $24 \mathrm{~h}$ at $37^{\circ} \mathrm{C}$. After incubation, a TUNEL assay was performed. Scale bar $=50 \mu \mathrm{m}$.

cells and poured $1.5 \mathrm{ml}$ of $0.36 \%$ agar into the 6 -well plate as top agar which was mixed in each well. We incubated the cells for 1 or 2 weeks and counted the number of colonies.

Statistical analysis. Statistical analysis was performed using Student's $t$ test and one-way analysis of variance with GraphPad Prism 5 software (GraphPad Software, La Jolla, CA). The data are expressed as the means $\pm \mathrm{SE}$ of the mean $(n=3-6)$ unless otherwise specified. All experiments were performed in triplicate $(* p<0.05, * * p<0.01, * * * p<0.001)$.

\section{Results}

The killing effect of NTP is specific to squamous cell carcinoma cells. We performed a cell viability assay to evaluate the effect of NTP and compared differences in the effect between fibroblasts and squamous cell carcinoma cells. The results showed that the viability of HS-K and IMR-SV-90 cells did not significantly decrease upon NTP application until $120 \mathrm{~s}$. However, the cell viability of the squamous cell carcinoma cell lines began to rapidly and significantly decrease with increasing NTP application time (Fig. 1A). SAS, HSC-3 and HO-1-u-1 cells are poorly differentiated cancer cells, whereas the other squamous cell carcinoma cells are well differentiated. The average application time leading to a significant decrease in cell survival was $100 \mathrm{~s}$ and $75 \mathrm{~s}$, respectively, for each differentiation group. The exposure time until cell death by NTP for the well-differentiated cancer cells tended to be shorter than that for the poorly differentiated cancer cells. To evaluate cell death type of oral squamous cell carcinoma, we measured Annexin V-FITC and propidium iodide (PI) staining. We found that apoptosis occurred after NTP application to SAS 

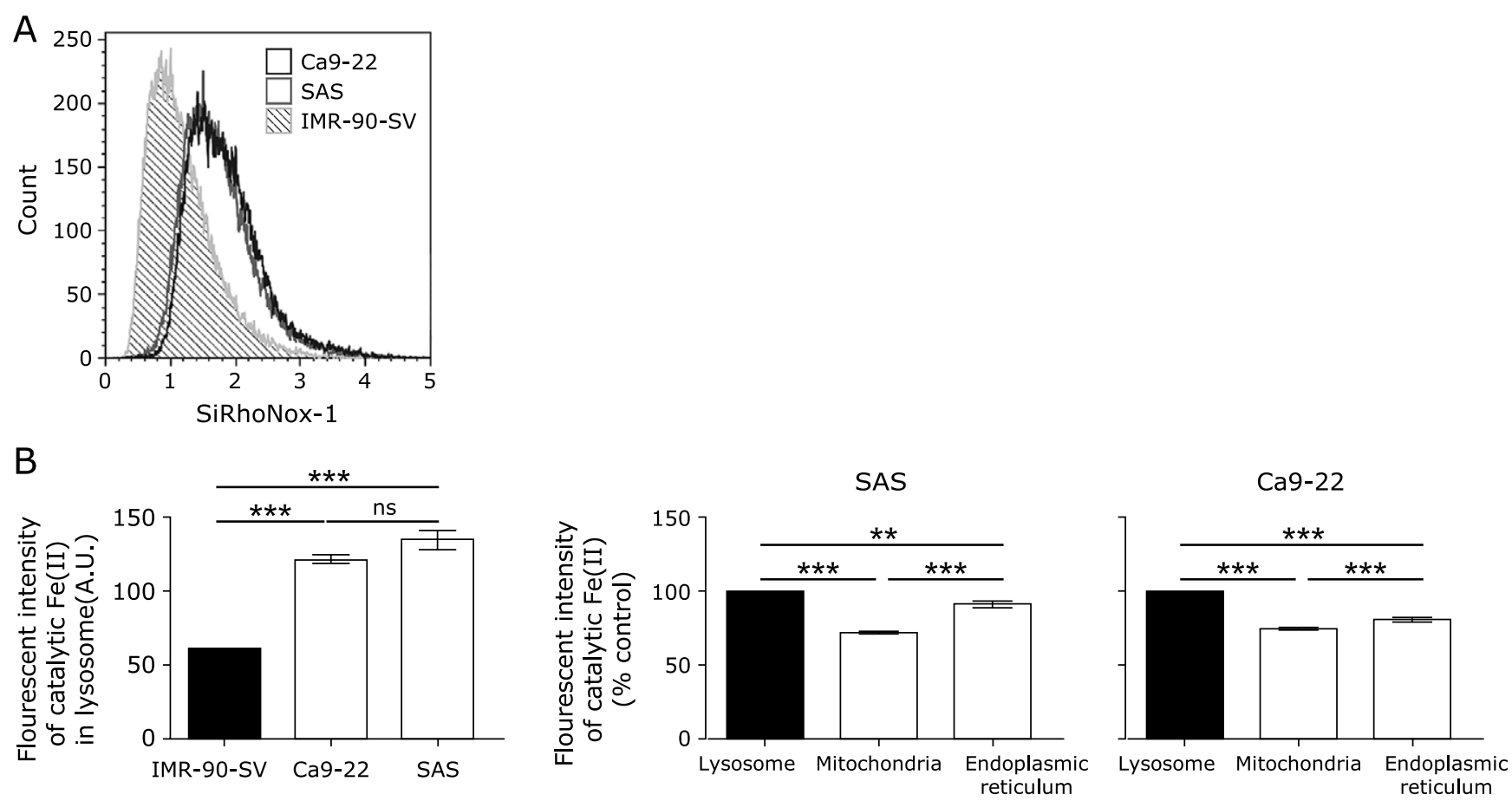

C

SiRhoNox-1

Lysosome

Mitochondria
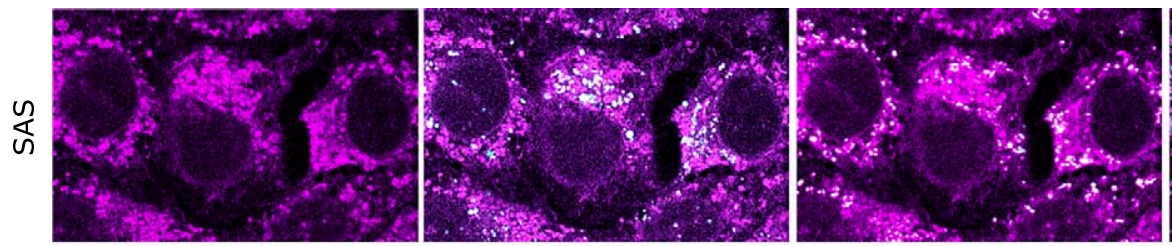

Endoplasmic reticulum
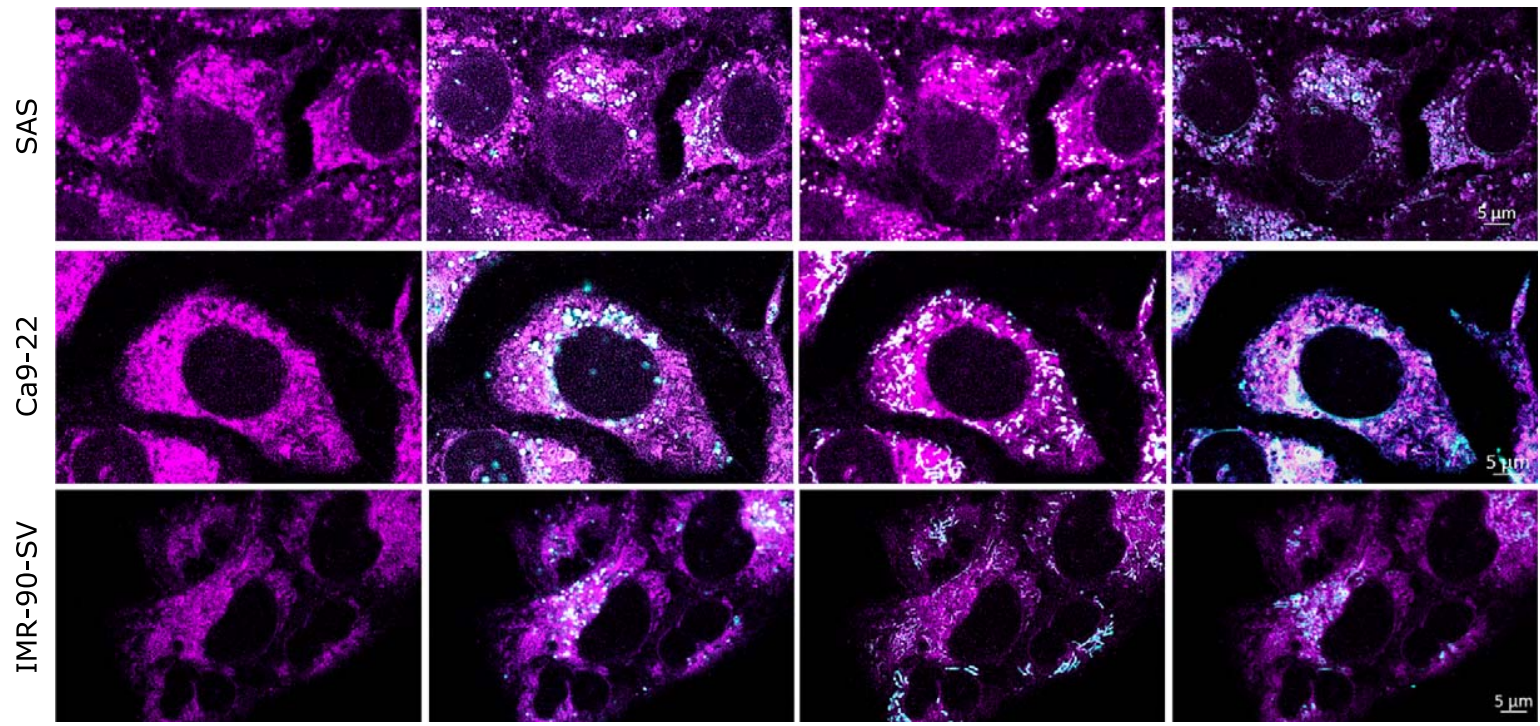

Fig. 2. Squamous cell carcinoma cells contain more catalytic Fe(II) than fibroblast cells. (A) $5 \times 10^{5}$ cells were stained with SiRhoNox- $1(1 \mu \mathrm{M})$, and the fluorescence intensity of individual cells was evaluated using flow cytometry. (B) A total of $2.5 \times 10^{5}$ cells were plated into glass-bottom dishes and incubated for $24 \mathrm{~h}$ at $37^{\circ} \mathrm{C}$. Then, the cells were stained with SiRhoNox-1 (1 $\left.\mu \mathrm{M}\right)$, MitoTracker Green FM (50 nM), ER-Tracker Blue-White DPX $(200 \mathrm{nM})$ and LysoTracker Red DND-99 $(200 \mathrm{nM})$, and the cells were observed using a confocal microscope. Scale bar $=5 \mu \mathrm{m}$. Error bars represent the standard error of the mean $(n>20) . * * p<0.01, * * * p<0.001 ; n s$, not significant.

and Ca9-22 cells (Fig. 1B). In addition, according to the TUNEL assay, we also observed apoptotic cells in the NTP group (Fig. 1C).

Squamous cell carcinoma cells harbor more catalytic Fe(II) than fibroblast cells. To study the association between Fe and NTP cytotoxicity, we measured catalytic Fe(II) in individual cells using SiRhoNox-1. We observed that the Fe level in IMR-SV-90 fibroblast cells was lower than those in SAS and Ca9-22 cancer cells; however, no difference in the iron levels were observed between SAS and Ca9-22 cells according to the flow cytometric analysis (Fig. 2A). Consequently, to evaluate the localization of catalytic Fe(II), we observed the cells, using confocal microscopy. This examination revealed that the intensity of catalytic Fe(II) in the lysosomes of IMR-SV-90 cells was lower than that in SAS and Ca9-22 lysosomes (Fig. 2B). Furthermore, we found that the intensity of catalytic $\mathrm{Fe}$ (II) in lysosomes of cancer cells was higher than that in the mitochondria and endoplasmic reticulum (ER).

The killing effects of NTP depend on the amounts of catalytic Fe(II). We examined differences in the killing effect of NTP by modifying the intracellular iron level in each cell. SAS and Ca9-22 cells were loaded by FAC, and then the catalytic 

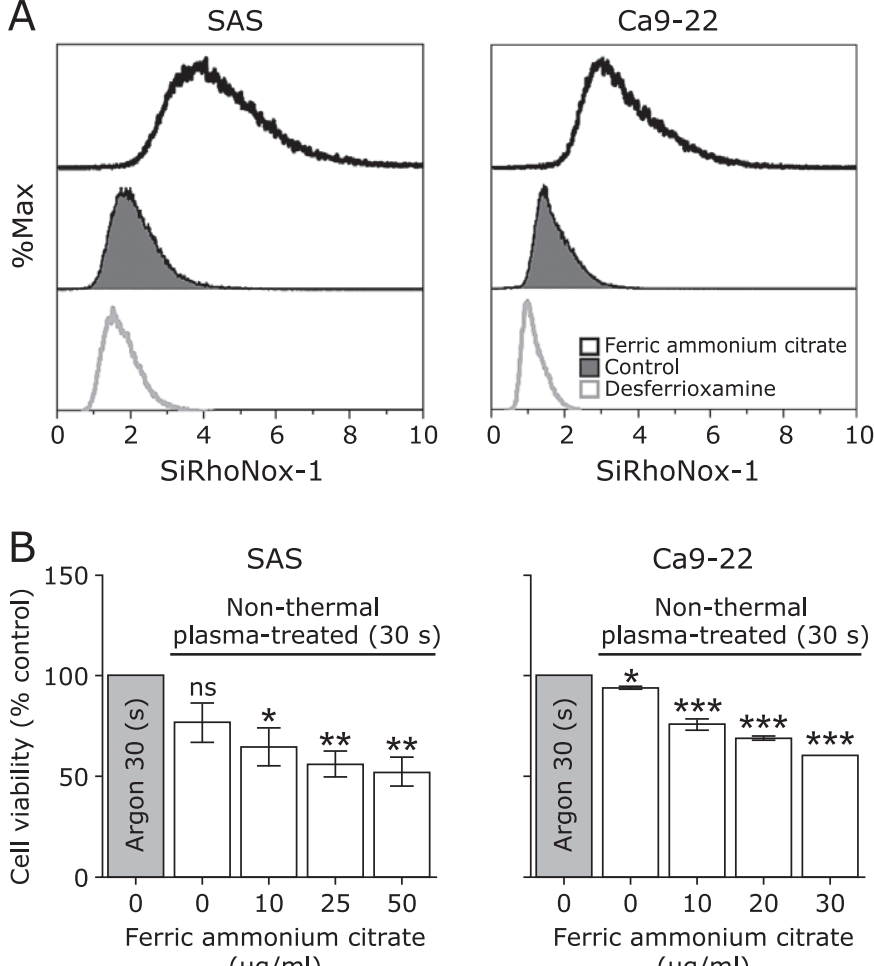

$(\mu \mathrm{g} / \mathrm{ml})$
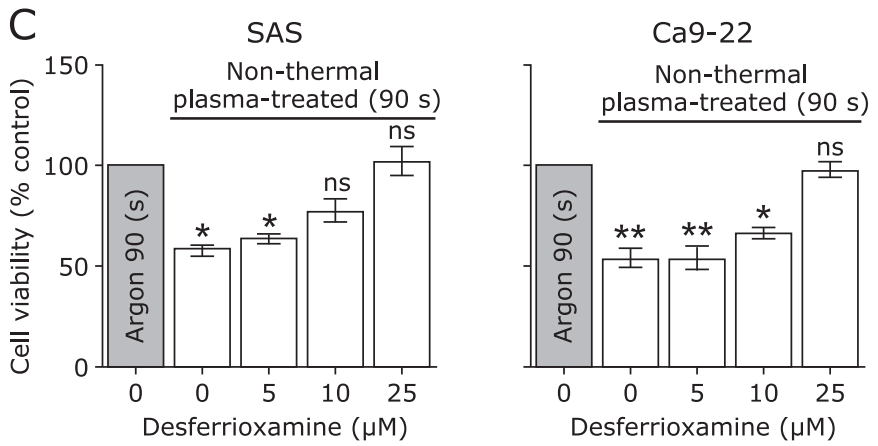

Fig. 3. The effect of non-thermal plasma (NTP) changes depending on the amount of catalytic Fe(II). (A) SAS and Ca9-22 cells were seeded in $60-\mathrm{mm}$ dishes at $5.0 \times 10^{5}$ cells for $24 \mathrm{~h}$. The cells were loaded for $3 \mathrm{~h}$ with ferric ammonium citrate (FAC) $(10 \mu \mathrm{g} / \mathrm{ml})$ and desferrioxamine (DFO) $(25 \mu \mathrm{M})$ in each group. Then, the cells were stained with SiRhoNox-1 $(1 \mu \mathrm{M})$ for $30 \mathrm{~min}$. The fluorescence intensity of each cell was evaluated using flow cytometry. (B) SAS and Ca 9-22 cells were cultured in 96 -well plates at 5,000 cells/well for $24 \mathrm{~h}$ at $37^{\circ} \mathrm{C}$. The cells were loaded for $3 \mathrm{~h}$ with FAC. Then, NTP was applied for $30 \mathrm{~s}$, and the cells were cultured for $24 \mathrm{~h}$ at $37^{\circ} \mathrm{C}$. Absorbance was measured. (C) The cells were loaded with DFO for $3 \mathrm{~h}$. Then, NTP was applied for $90 \mathrm{~s}$, and the cells were cultured for $24 \mathrm{~h}$ at $37^{\circ} \mathrm{C}$. Absorbance was measured. Error bars represent the standard error of the mean. ${ }^{*} p<0.05,{ }^{*} p<0.01$, $* * * p<0.001 ; \mathrm{ns}$, not significant.

$\mathrm{Fe}(\mathrm{II})$ levels increased in both cell types. Conversely, when DFO was applied, the catalytic Fe(II) levels decreased in both cell types (Fig. 3A). Subsequently, we evaluated the effect of iron modification at NTP application. The results revealed that the viability of SAS and Ca9-22 cells significantly decreased as the applied concentration of FAC increased (Fig. 3B). Conversely, the viability of SAS and Ca9-22 cells significantly increased as the applied concentration of DFO increased (Fig. 3C).
NTP exposure increases ROS in squamous cell carcinoma cells. To evaluate ROS generation after applying NTP to squamous cell carcinoma cells, we used DCF, MitoSOX and BODIPY. DCF has been reported to detect various cytoplasmic peroxides, MitoSOX detects mitochondrial superoxide, and BODIPY reports membrane lipid peroxidation. After applying NTP, the level of ROS significantly increased for all the probes (Fig. 4)

NTP suppresses the migration and invasion activities of squamous cell carcinoma cells. To examine migration ability, we performed a scratch wound-healing assay and a transwell migration assay. The results revealed that NTP application suppressed the migratory ability of SAS and $\mathrm{Ca} 9-22$ cells compared to Ar alone, as shown in both assays (Fig. 5A and B). To evaluate the invasive nature of squamous cell carcinoma cells, we performed a transwell invasion assay. The results showed that NTP exposure suppressed the invasive ability of SAS and Ca9-22 cells compared with Ar alone (Fig. 5C). According to the Western blotting, the protein level of MMP-2 decreased in NTP group compared with Ar group (Supplemental Fig. 1*). Subsequently, the anchorage-independent proliferation of squamous cell carcinoma cells was assessed with a colony formation assay. The results revealed that for the SAS cell line, the Ar alone group showed significant anchorage-independent growth compared with the NTP group. However, in the case of the Ca9-22 cell line, colonies were not established in either the NTP or Ar alone group (Fig. 5D).

\section{Discussion}

Here, we studied the effects of NTP exposure on oral squamous cell carcinoma for the first time, with a focus on catalytic Fe(II). A distinct difference in the effects of NTP was observed between the oral cancer cells and non-tumorous cells. We found a proportional association between the killing effect of NTP exposure and the amounts of catalytic Fe(II) in oral squamous cell carcinoma cells in iron modification experiments.

Oxidative stress via excess iron has been associated with carcinogenesis, ${ }^{(20)}$ and cancer cells are oxidatively stressed in general. ${ }^{(35)}$ In a previous experiment, we showed that cancer cells have more catalytic Fe(II) through maturation of HL-60 leukemia cells to macrophage-like cells. ${ }^{(34)}$ Here we revealed that abundance of catalytic $\mathrm{Fe}(\mathrm{II})$ was also true for the oral squamous cell carcinoma cells, with a higher fraction in the lysosomes than in the mitochondria and ER. In this experiment, we did not find a difference in the levels of catalytic Fe(II) between well-differentiated and poorly-differentiated oral cancer cells. However, we believe that this finding is the basis for the differential effect of NTP between tumor and non-tumor cells.

Regarding the type of cell death induced by NTP, we obtained a few evidences supporting apoptosis; namely, increased annexin V with flow cytometric analysis and TUNEL positivity. However, simultaneously, we observed iron-dependent lipid peroxidation and mitochondrial superoxide during cell death by the use of three different fluorescent probes, thus suggesting the mixed presence of ferroptotic process. In the case of mesothelioma cells exposed to NTP, we observed activation of the autophagic pathway and lipid peroxidation, suggesting ferroptosis. ${ }^{(19)}$ On the contrary, ovarian cancer and melanoma skin cancer cells mainly showed apoptosis upon NTP exposure. ${ }^{(17,36)}$ Therefore, the cell death type may be different among different cancer cells. Whether we can clearly differentiate between apoptosis and ferroptosis with the current techniques is an intriguing issue, which certainly requires further investigation, including development of novel methods to detect ferroptosis.

Due to the accessibility of the oral cavity, clinical application of NTP is easier at this site compared to other organs, except for the skin. The depth of penetration with direct NTP exposure is $\sim 1 \mathrm{~mm} .{ }^{(15)}$ We also showed that NTP treatment of oral cancer cells 

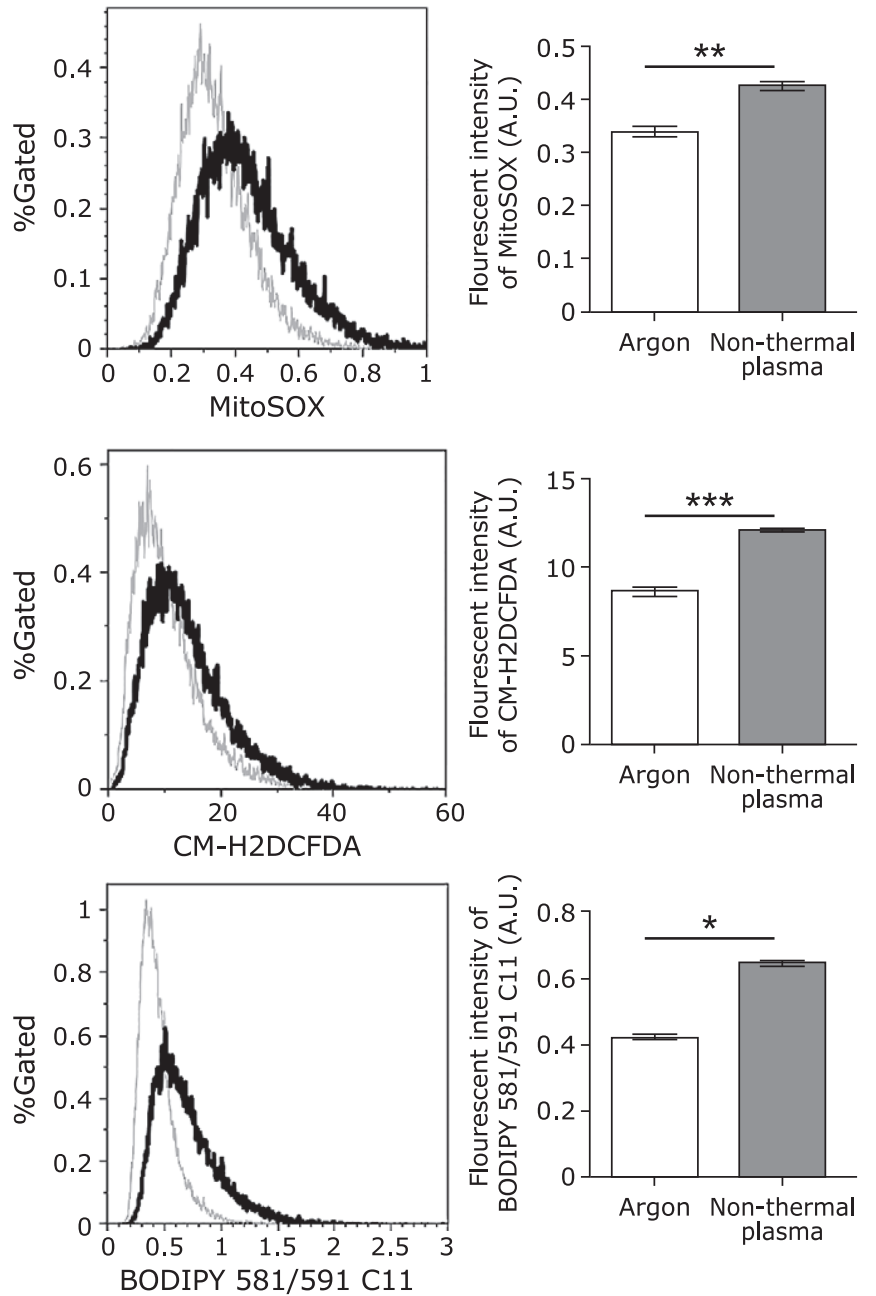
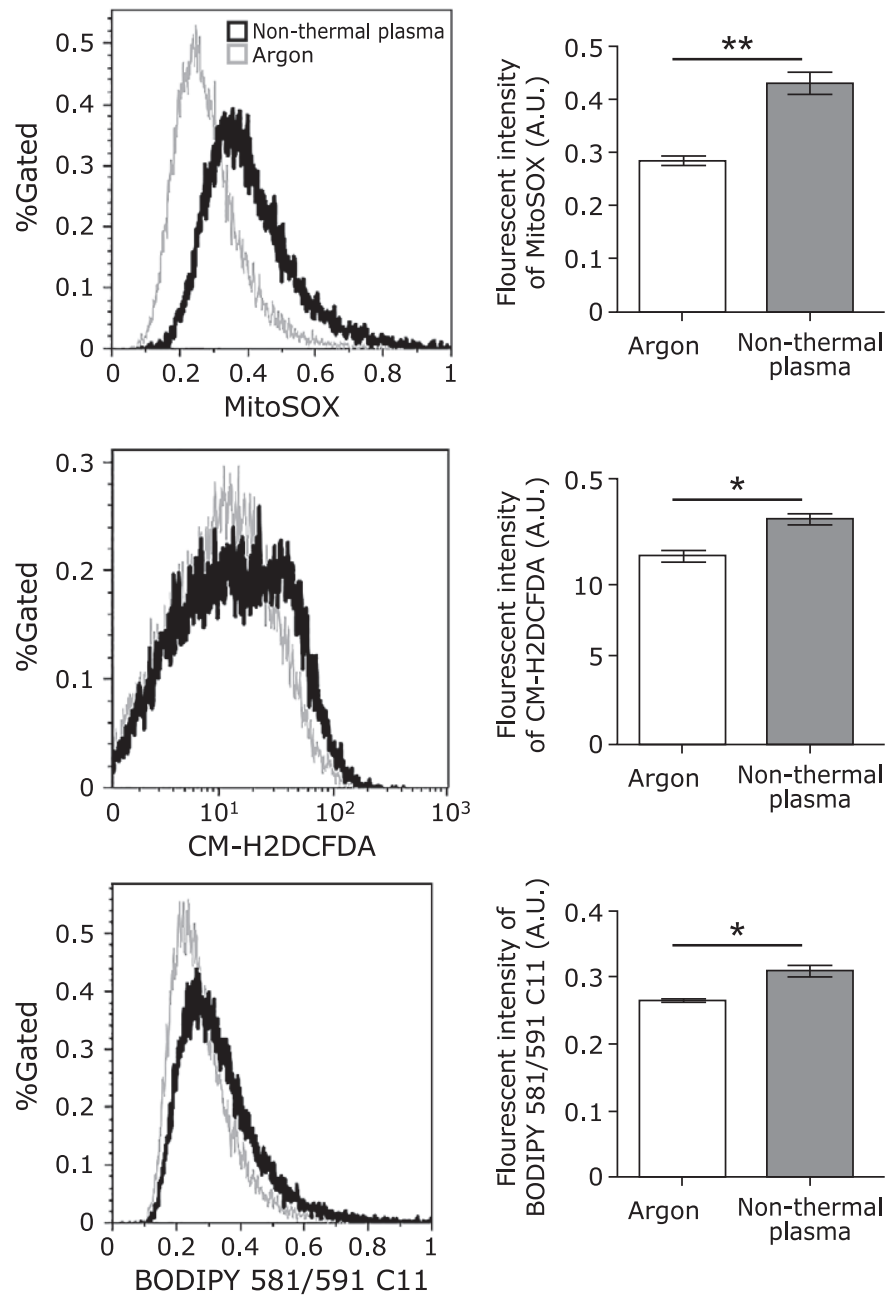

Fig. 4. Non-thermal plasma results in reactive oxygen species (ROS) generation. ROS generation was detected using MitoSOX (5 $\mu \mathrm{M})$, a chloromethyl derivative of $2^{\prime}, 7^{\prime}$-dichlorodihydrofluorescein (DCF) $(10 \mu \mathrm{M})$ and BODIPY $581 / 591$ C11 (BODIPY) (2 $\left.\mu \mathrm{M}\right)$ by flow cytometry. All signals significantly increased. Error bars represent the standard error of the mean. ${ }^{*} p<0.05, * * p<0.01,{ }^{* *} p<0.001$; ns, not significant.

significantly impairs tumor migration and invasion, which may at least result from suppressed MMP-2 expression. As clinical applications, NTP can be used for preoperative progression control and irradiation of intraoperative margins rather than curative treatment. NTP treatment may be suitable for preventing recurrence after surgery. In general, a $10-\mathrm{mm}$ safety margin is used in oral surgery. However, if this large margin can be reduced with NTP exposure, then functional loss can be reduced, resulting in cosmetic benefits and better quality of life. Notably, NTP exposure is substantially less harmful to normal tissues, as shown by the findings in fibroblasts, and can further promote wound healing. ${ }^{(18,37)}$ We believe that NTP exposure as an additional therapy is feasible until radiation therapy and chemotherapy are completed. Preclinical in vivo experiments are in progress to study the merits of NTP exposure.

In conclusion, NTP exposure specifically killed oral squamous carcinoma cells compared to fibroblasts, which was dependent on ample catalytic Fe(II). The type of cell death was mixed apoptosis and ferroptosis.

\section{Acknowledgments}

We would like to thank Norihisa Ichimura and Fumiya Kano for their detailed comments, suggestions and constant support. This work was supported in part by a JSPS KAKENHI (Grant Number JP17H04064; JP16K15257; JP24108008) and the Private University Research Branding Project to ST.

\section{Abbreviations}

\begin{tabular}{|c|c|}
\hline Ar & argon \\
\hline BODIPY & BODIPY 581/591 C11 \\
\hline $\mathrm{CF}$ & in \\
\hline DFO & desferrioxamine (desferal) \\
\hline ER & lasmic reticulum \\
\hline FAC & ammonium citrate \\
\hline FITC & fluorescein isothiocyanate \\
\hline FBS & fetal bovine serum \\
\hline MMP-2 & matrix metalloproteinase- 2 \\
\hline NTP & hermal plasma \\
\hline PR & phosphate-buffered saline \\
\hline
\end{tabular}



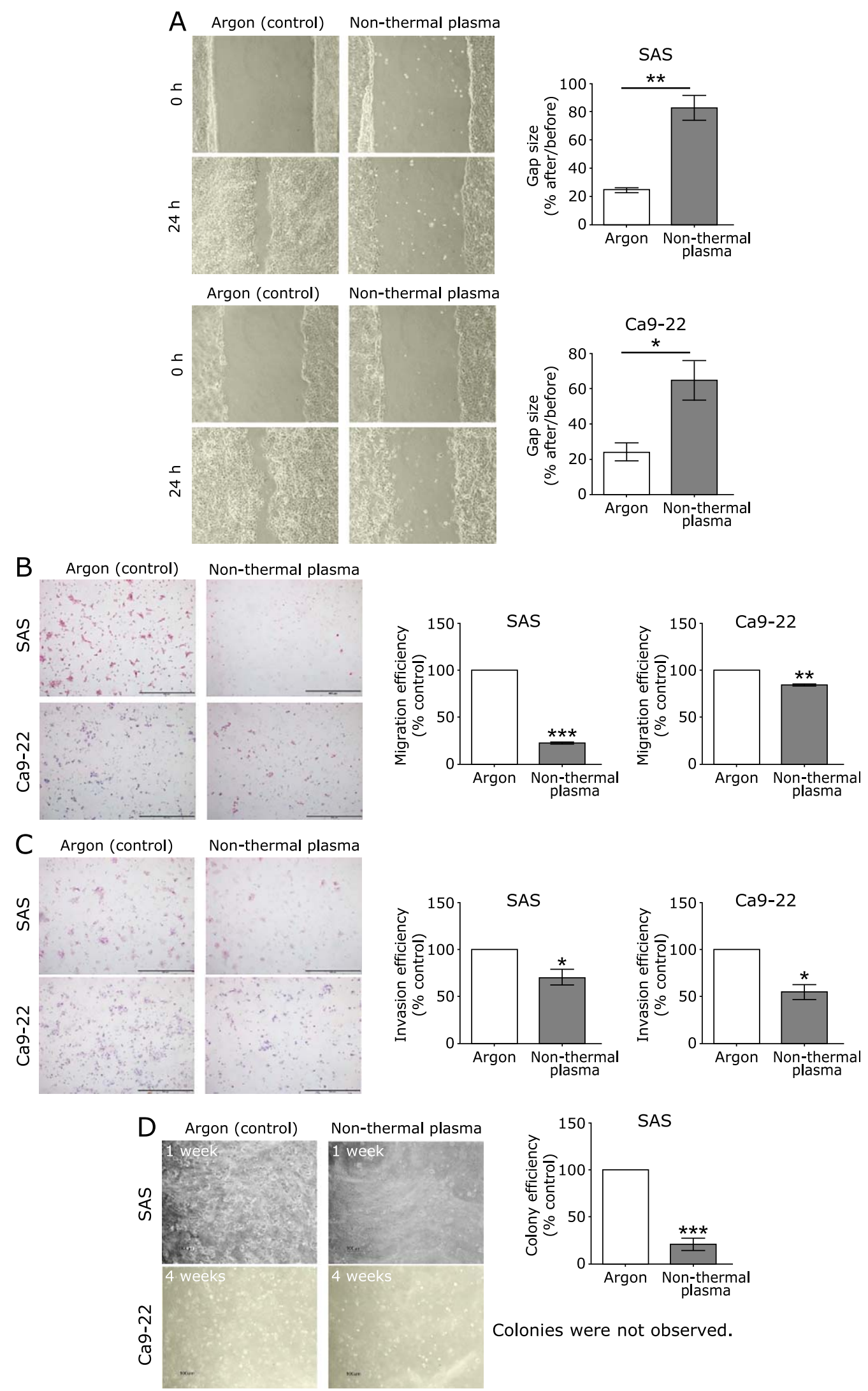

Colonies were not observed.

Fig. 5. Non-thermal plasma suppresses the migration and invasion abilities of squamous cell carcinoma cells. (A) Wound-healing assay at 0 and $24 \mathrm{~h}$ after treatment. (B, C) Transwell migration and invasion assays at $24 \mathrm{~h}$ after treatment. Five representative fields of cells in each group were captured and analyzed. Scale bar $=500 \mu \mathrm{m}$. (D) Colony formation assay. Four representative fields of cells in each group were captured and analyzed. Scale bar $=500 \mu \mathrm{m}$. Error bars represent the standard error of the mean. ${ }^{\star} p<0.05,{ }^{*} p<0.01,{ }^{*} * p<0.001 ;$ ns, not significant. 
UV terminal deoxynucleotidyl transferase-mediated dUTP nick end labeling ultraviolet

\section{Conflict of interest}

No potential conflicts of interest were disclosed.

\section{References}

1 Jemal A, Bray F, Center MM, Ferlay J, Ward E, Forman D. Global cancer statistics. CA Cancer J Clin 2011; 61: 69-90.

2 Kim HY, Elter JR, Francis TG, Patton LL. Prevention and early detection of oral and pharyngeal cancer in veterans. Oral Surg Oral Med Oral Pathol Oral Radiol Endod 2006; 102: 625-631.

3 Blot WJ, McLaughlin JK, Winn DM, et al. Smoking and drinking in relation to oral and pharyngeal cancer. Cancer Res 1988; 48: 3282-3287.

4 Hashibe M, Brennan P, Chuang SC, et al. Interaction between tobacco and alcohol use and the risk of head and neck cancer: pooled analysis in the International Head and Neck Cancer Epidemiology Consortium. Cancer Epidemiol Biomarkers Prev 2009; 18: 541-550.

5 Hori M, Choi EH, Toyokuni S. Low-temperature plasma in biology and medicine. Arch Biochem Biophys 2016; 605: 1-2.

6 Kaneko T, Sasaki S, Takashima K, Kanzaki M. Gas-liquid interfacial plasmas producing reactive species for cell membrane permeabilization. J Clin Biochem Nutr 2017; 60: 3-11.

7 Tanaka H, Mizuno M, Ishikawa K, et al. Plasma with high electron density and plasma-activated medium for cancer treatment. Clin Plasma Med 2015; 3: $72-76$.

8 Moreau M, Orange N, Feuilloley MG. Non-thermal plasma technologies: new tools for bio-decontamination. Biotechnol Adv 2008; 26: 610-617.

9 Fridman G, Fridman G, Gutsol A, Shekhter AB, Vasilets VN, Fridman A. Applied plasma medicine. Plasma Process Polym 2008; 5: 503-533.

10 Yasuda H, Miura T, Kurita H, Takashima K, Mizuno A. Biological evaluation of DNA damage in bacteriophages inactivated by atmospheric pressure cold plasma. Plasma Process Polym 2010; 7: 301-308.

11 Vandamme M, Robert E, Lerondel S, et al. ROS implication in a new antitumor strategy based on non-thermal plasma. Int J Cancer 2012; 130: 2185 2194.

12 Brullé L, Vandamme M, Riès D, et al. Effects of a non thermal plasma treatment alone or in combination with gemcitabine in a MIA PaCa2-luc orthotopic pancreatic carcinoma model. PLoS One 2012; 7: e52653.

13 Utsumi F, Kajiyama H, Nakamura K, et al. Effect of indirect nonequilibrium atmospheric pressure plasma on anti-proliferative activity against chronic chemo-resistant ovarian cancer cells in vitro and in vivo. PLoS One 2013; 8: e81576.

14 Ishaq M, Evans MM, Ostrikov KK. Effect of atmospheric gas plasmas on cancer cell signaling. Int J Cancer 2014; 134: 1517-1528.

15 Okazaki Y, Wang Y, Tanaka H, et al. Direct exposure of non-equilibrium atmospheric pressure plasma confers simultaneous oxidative and ultraviolet modifications in biomolecules. J Clin Biochem Nutr 2014; 55: 207-215.

16 Isbary G, Morfill G, Schmidt HU, et al. A first prospective randomized controlled trial to decrease bacterial load using cold atmospheric argon plasma on chronic wounds in patients. Br J Dermatol 2010; 163: 78-82.

17 Iseki S, Nakamura K, Hayashi M, et al. Selective killing of ovarian cancer cells through induction of apoptosis by nonequilibrium atmospheric pressure plasma. Appl Phys Lett 2012; 100: 113702.

18 Miyamoto K, Ikehara S, Sakakita H, Ikehara Y. Low temperature plasma equipment applied on surgical hemostasis and wound healings. J Clin Biochem Nutr 2017; 60: 25-28.

19 Shi L, Ito F, Wang Y, et al. Non-thermal plasma induces a stress response in mesothelioma cells resulting in increased endocytosis, lysosome biogenesis and autophagy. Free Radic Biol Med 2017; 108: 904-917.

20 Toyokuni S. Role of iron in carcinogenesis: cancer as a ferrotoxic disease. Cancer Sci 2009; 100: 9-16.

21 Toyokuni S. Iron as a target of chemoprevention for longevity in humans. Free Radic Res 2011; 45: 906-917.

22 Jiang L, Akatsuka S, Nagai H, et al. Iron overload signature in chrysotileinduced malignant mesothelioma. J Pathol 2012; 228: 366-377.

23 Akatsuka S, Yamashita Y, Ohara H, et al. Fenton reaction induced cancer in wild type rats recapitulates genomic alterations observed in human cancer. PLoS One 2012; 7: e43403.

24 Toyokuni S. The origin and future of oxidative stress pathology: from the recognition of carcinogenesis as an iron addiction with ferroptosis-resistance to non-thermal plasma therapy. Pathol Int 2016; 66: 245-259.

25 Stockwell BR, Friedmann Angeli JP, Bayir H, et al. Ferroptosis: a regulated cell death nexus linking metabolism, redox biology, and disease. Cell 2017; 171: 273-285.

26 Nagai H, Okazaki Y, Chew SH, Misawa N, Yasui H, Toyokuni S. Deferasirox induces mesenchymal-epithelial transition in crocidolite-induced mesothelial carcinogenesis in rats. Cancer Prev Res (Phila) 2013; 6: 1222-1230.

27 Ohara Y, Chew SH, Shibata T, et al. Phlebotomy as a preventive measure for crocidolite-induced mesothelioma in male rats. Cancer Sci 2018; 109: 330339.

28 Zacharski LR, Chow BK, Howes PS, et al. Decreased cancer risk after iron reduction in patients with peripheral arterial disease: results from a randomized trial. J Natl Cancer Inst 2008; 100: 996-1002.

29 Chang JW, Kang SU, Shin YS, et al. Non-thermal atmospheric pressure plasma induces apoptosis in oral cavity squamous cell carcinoma: involvement of DNA-damage-triggering sub-G(1) arrest via the ATM/p53 pathway. Arch Biochem Biophys 2014; 545: 133-140.

30 Kang SU, Seo SJ, Kim YS, et al. Comparative effects of non-thermal atmospheric pressure plasma on migration and invasion in oral squamous cell cancer, by gas type. Yonsei Med J 2017; 58: 272-281.

31 Tanaka H, Mizuno M, Ishikawa K, et al. Plasma-activated medium selectively kills glioblastoma brain tumor cells by downregulating a survival signaling molecule, AKT kinase. Plasma Med 2011; 1: 265-277.

32 Hirayama T, Tsuboi H, Niwa M, et al. A universal fluorogenic switch for $\mathrm{Fe}$ (ii) ion based on $\mathrm{N}$-oxide chemistry permits the visualization of intracellular redox equilibrium shift towards labile iron in hypoxic tumor cells. Chem Sci 2017; 8: 4858-4866.

33 Ohara Y, Chew SH, Misawa N, et al. Connective tissue growth factor-specific monoclonal antibody inhibits growth of malignant mesothelioma in an orthotopic mouse model. Oncotarget 2018; 9: 18494-18509.

34 Ito F, Nishiyama T, Shi L, et al. Contrasting intra- and extracellular distribution of catalytic ferrous iron in ovalbumin-induced peritonitis. Biochem Biophys Res Commun 2016; 476: 600-606.

35 Toyokuni S, Okamoto K, Yodoi J, Hiai H. Persistent oxidative stress in cancer. FEBS Lett 1995; 358: 1-3.

36 Fridman G, Shereshevsky A, Monika M, et al. Floating electrode dielectric barrier discharge plasma in air promoting apoptotic behavior in melanoma skin cancer cell lines. Plasma Chem Plasma Process 2007; 27: 163-176.

37 Choi JH, Song YS, Song K, Lee HJ, Hong JW, Kim GC. Skin renewal activity of non-thermal plasma through the activation of beta-catenin in keratinocytes. Sci Rep 2017; 7: 6146 